\title{
Using Convolutional Neural Network for Edge Detection in Musculoskeletal Ultrasound Images
}

\author{
Shaima I. Jabbar, Charles R. Day Member, IEEE, Nicholas Heinz and Edward K. Chadwick
}

\begin{abstract}
Fast and accurate segmentation of musculoskeletal ultrasound images is an on-going challenge. Two principal factors make this task difficult: firstly, the presence of speckle noise arising from the interference that accompanies all coherent imaging approaches; secondly, the sometimes subtle interaction between musculoskeletal components that leads to non-uniformity of the image intensity. Our work presents an investigation of the potential of Convolutional Neural Networks (CNNs) to address both of these problems. CNNs are an effective tool that has previously been used in image processing of several biomedical imaging modalities. However, there is little published material addressing the processing of musculoskeletal ultrasound images, particularly using a panoramic technique. In our work we explore the effectiveness of CNNs when trained to act as a pre-segmentation pixel classifier that determines whether a pixel is an edge or non-edge pixel. Our CNNs are trained using two different ground truth interpretations. The first one uses an automatic Canny edge detector to provide the ground truth image; in the second interpretation, the ground truth was obtained using the same image marked-up by an expert anatomist. In this initial study the CNNs have been trained using half of the prepared data from one image, using the other half for testing; validation was also carried out using three unseen ultrasound images. CNN performance was assessed using Mathew's Correlation Coefficient, Sensitivity, Specificity and Accuracy. The results show that CNN performance when using expert ground truth image is better than in the case of using Canny ground truth image. Our technique is promising and has the potential to speed-up the image processing pipeline using appropriately trained CNNs.
\end{abstract}

Index Terms - Segmentation, Convolutional Neural Networks, Musculoskeletal model, Ultrasound image

\section{INTRODUCTION}

$\mathrm{M}$ usculoskeletal Ultrasound Imagery (MUI) arises from the response of the sound waves that comes from ultrasonography scanning of muscle, tendon, bone and other musculoskeletal components. It is a flexible imaging modality because not only is it free from ionizing radiation and magnetic fields, but it is also very cost effective and highly portable. Also, imaging via ultrasonography can be performed in real-time; it is non-

S. I. Jabbar, is Ph.D. student in biomedical Engineering, Institute for Science and Technology in Medicine, Keele University, UK (s.i.jabbar@keele.ac.uk)

C.R. Day, lecturer in Computer Science, Keele University, UK (c.r.day@ keele.ac.uk)

N. Heinz, has MMedSci in Anatomical Sciences, Keele University, UK (v1e52@students.keele.ac.uk)

E. K., Chadwick is lecturer in Biomedical Engineering, Institute for Science and Technology in Medicine Keele University, UK (e.k.j.chadwick@keele.ac.uk invasive and interestingly allows dynamic free movement. However, conventional ultrasonography has limitations due to a relatively narrow field of view. The panoramic ultrasound imaging technique has arisen to extend the field view of the ultrasound imagery [1]. In the panoramic imaging technique, a transducer collects a panorama of images as a rapid succession of frames: keeping the old frame and continues to add a newly scanned image to the previous ones. All scanned frames are parallel to the direction of scan plane. In this way the ultrasonographer can elongate the field of view of the image. So, this is a powerful technique that potentially allows the clinician to see a whole muscle from origin to insertion at one instance. This kind of accurate muscle imaging will help in the estimation of important geometric parameters that are used in computational musculoskeletal models: such as muscle fascicle length and tendon length in the long muscles.

The main purpose of computational musculoskeletal modelling is the analysis of human movement and skeletal loading resulting from internal forces in the musculoskeletal system in vivo [2]. Fundamental to the usefulness of musculoskeletal modelling is the accurate determination of these kinds of geometric parameters. 
Estimates of these parameter values have previously been obtained from cadavers [3], and then either treated as generic parameters of scaled based on external measurements. But subject-specific, automatic, close to real time, determination of these parameters when the MUI is captured is also highly desirable. That is considered essential to achieve personalized musculoskeletal model based on individual patient musculoskeletal characteristics. Figure 1 illustrates a typical scenario: starting from scanning the shoulder region by ultrasonography, to image processing which includes image enhancement, edge detection and segmentation. This allows us to extract the important parameters for the musculoskeletal model.

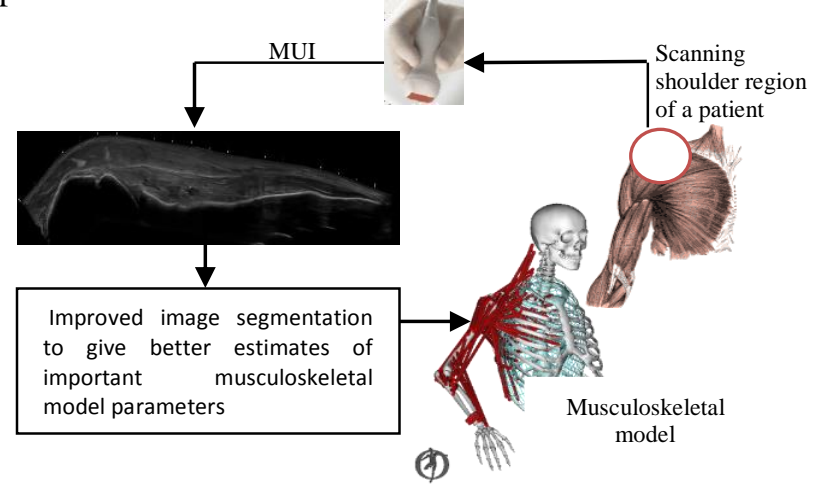

Fig. 1 shows the intended application of this work in the image processing pipeline, where images have been recorded from patient for processing: image enhancement, edge detection and segmentation.

Image processing and segmentation of MUI is not a trivial task due to speckle noise, and the low contrast and homogeneity of ultrasound gray level intensities [4]. The detection and elimination of speckle noise in ultrasound images is a challenge because it is multiplicative noise. The main reason is the coherent nature of the ultrasound image acquisition process [5]. Multiplicative noise is more problematic than additive noise since it is not amenable to simple linear filtering [6]. There is little doubt that despeckling ultrasound images would enhance the performance of image classifiers such as our CNNs. However, in this initial study we want to see how well the noise toleration properties of CNNs are able to cope with this noise.

The first automatic segmentation technique on MUI was in 2014 [4]. This technique addressed at the beginning some issues related with MUI such as speckle noise reduction and contrast enhancement. Extraction of image features was achieved by using a curvelet transform. Then the following steps used morphological processing like erosion and dilation to eliminate distorted pixels. Subsequent automatic segmentation was expected to support MUI diagnosis, but the muscle edges were not well preserved due to dilation and erosion. Furthermore, the MUI was not obtained using the processing constraints introduced by the panoramic technique, and it was not performed on those muscle groups that we are attempting to label as shown in figure $2 \mathrm{~d}$, below.

Convolutional Neural Networks (CNNs) are one member of the Deep Learning Neural Network (DNN) family. The simple concept of CNN is inherited from a biological process at the visual cortex. CNN consists of successions of different types of layers including convolutional layers, subsampling layers and finally a fully connected classification layer [7]. CNNs have been used in many different types of biomedical imaging applications. One of these applications is the classification of a pixel as a membrane or not in electron microscopy biopsy images by Ciresan et. al.[8]. Ciresan's work made extensive use of graphics processing units (GPUs) in the training of their CNNs. GPU has a significant role in the acceleration of deep neural network training [9], but no special GPU architecture is required once the CNNs have been trained. In 2013, this scenario of pixel classification was also applied to detect mitosis in breast cancer images; features that were fed to the classifier came from a fusion of $\mathrm{CNN}$ features and handcrafted features [10]. This work was also concerned with identifying mitosis without the need for handcrafted features [9]. CNNs have also been applied to X-ray image processing applications. Here, CNNs have been used to detect bone [11]. Recently, segmentation of blood vessels has been introduced as another application of using CNNs on retinal photographs [12]. This shows that CNNs are finding increasing use in biomedical image processing applications.

In this work we plan to recruit the $\mathrm{CNN}$ to investigate its ability to classify whether MUI pixels are edge or nonedge pixel of muscles. Two different types of ground truth images are involved in the development of the CNNs. One set of ground truth was obtained from a human expert and other comes from commonly used automatic method of edge detection. We compare between the results to demonstrate the difference in $\mathrm{CNN}$ performance in these two cases. Additionally, we have observed how well CNN can tolerate low image contrast and speckle noise. In neither of our two scenarios was any special noise reduction or feature extraction preprocessing applied.

\section{METHODS}

This section describes the approach we have used in detail and is organized as follows: A. Data collection and preparation; B. CNN configuration, C.CNN training details; D. Visualization of output images from training and testing processes. E. Quantitative assessment using the training and testing data; and F. further final validation with additional MUI that was not part of the training/testing process. 


\section{A. Dataset preparation}

Data was collected from scanning the left and right shoulder regions of a cadaver (male, 74 years old) by ultrasonography. Using a LOGIQ e Bt12 instrument, at $12 \mathrm{MHz}$ and the scanning technique use was the panoramic technique. The image dimensions were initially 550x1024, but following simple cropping of the background, the dimensions became $178 \times 783$. Four sample images were collected, one of the four (sample1) was used for CNN training and testing. While the rest of the ultrasound image samples (sample2, sample3 and sample4) have been used in the final validation. Figures $2 \mathrm{a}, 2 \mathrm{~b}, 2 \mathrm{c}, 2 \mathrm{~d}$ show for sample1: the original ultrasound image, the result of applying an automatic Canny edgedetector to sample1 to provide a Canny Ground Truth (CGT) image, a human expert derived set of edges to be used as the Expert Ground Truth (EGT) image and manually segmented version of the EGT to more clearly delineate the interesting muscle groups, respectively. We train and test separate CNNs on the same input image (sample1) but with two different ground truth images as the target in each case.

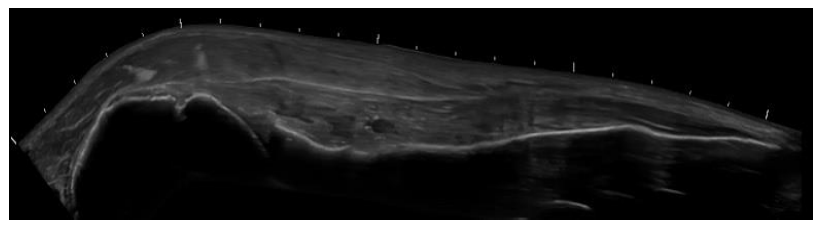

Fig .2a. Shows input image sample1

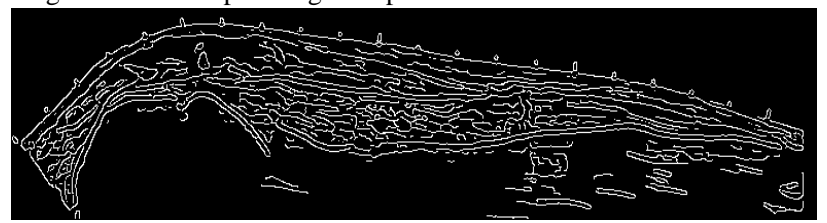

Fig.2c. shows CGT of sample1, it was extracted by using Matlab 8.6

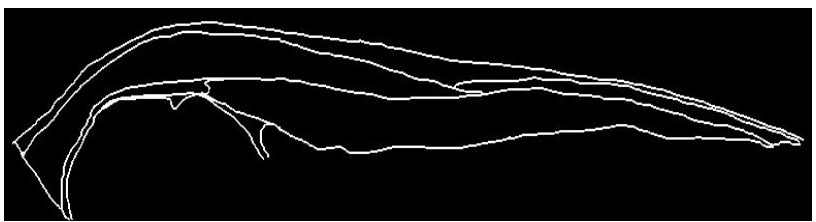

Fig. 2c. Shows EGT image, it was drawn by expert anatomist person

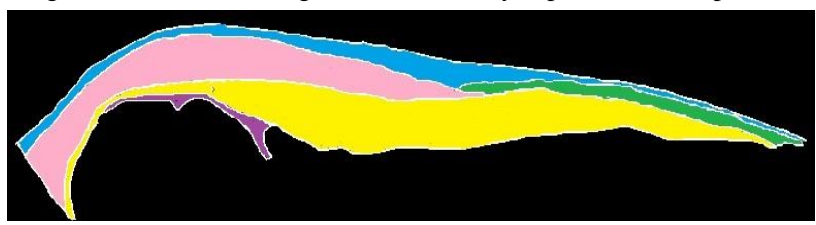

Fig. 2d. Illustrates Skin Trapezius muscle labeling of Fig. 2c. by Deltoid muscle Infraspinatus muscle

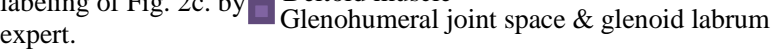

Initially, each pixel in the original image in Fig. 2a is labeled as edge pixel or non-edge pixel based on ground truth image and its $\mathrm{x}, \mathrm{y}$-coordinates are saved as well. The next step is doing a random selection of the labeled pixels where it is possible to track any pixel in the training and testing. In our work, 6000 and 10000 pixels have been selected randomly as edge and non-edge pixels respectively, so that the total pixels involved are 16000 . Training and testing datasets were chosen to have the same number of pixels ( 8000 pixels), and 8000 pixels of training data are disjoint from the testing pixels, so there is no overlap between the training and testing data (see Figure 3).

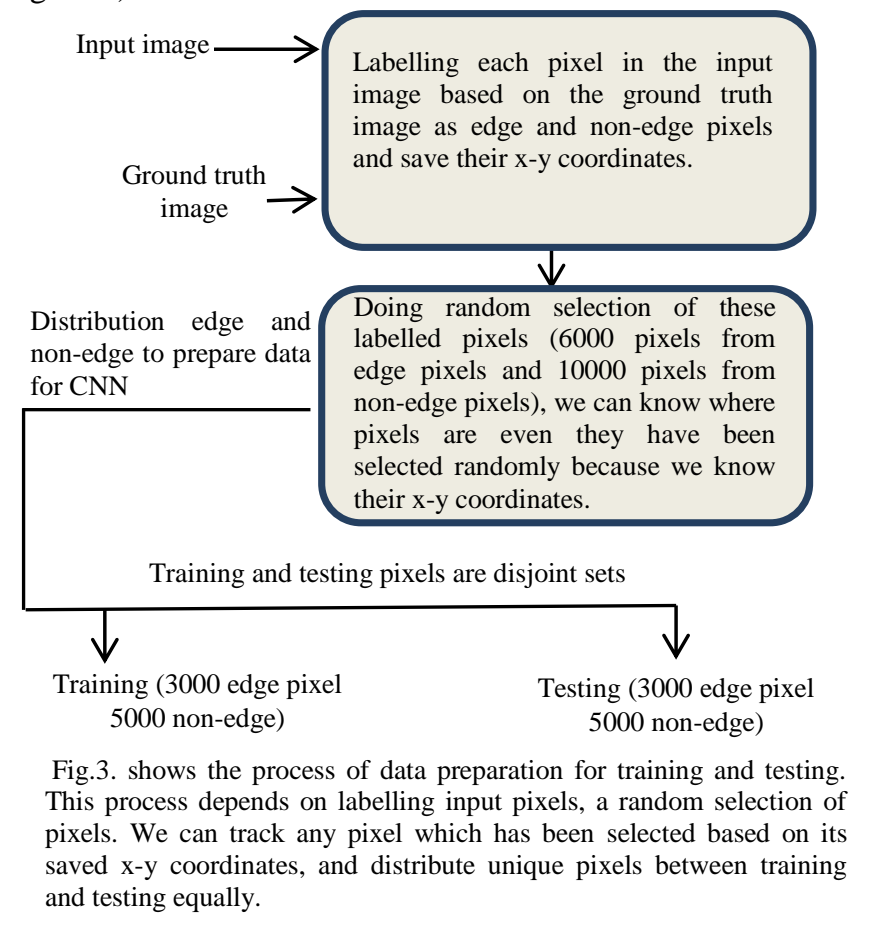

\section{B. CNN configuration}

Three main types of layers build the solid foundation of CNN processing. These layers are Convolutional Layers (CL), Max-pooling Layers (ML) and a final Fully Connected Layer (FCL). Some important problemspecific CNN parameters that must be carefully chosen include CNN feature-map kernel size (to avoid pixel fractions) and the overall number and size of the convolutional and max-pooling layers. Each of these has a powerful impact on the quality of the final outputs. So, several kernel sizes and numbers of feature maps in successive $\mathrm{CNN}$ layers have been evaluated for overall classification accuracy, see below. The FCL layer is a standard neural network classification layer [14]; the type of activation function used is a sigmoid function. It can be bounded within the range [minimum, maximum], so allows simple thresholding to get the final output classifications [15].

\section{C.Training}

All of this work was implemented in MATLAB 8.6. The training of each CNN used the input image from Fig. $2 \mathrm{a}$, but with separate ground truth images from Fig. $2 \mathrm{~b}$ and Fig 2c. The training was done on a computer with an Intel core i5 processer $(2.5 \mathrm{GHz}), 6 \mathrm{~GB}$ RAM and without GPU support. The training dataset preparation will vary slightly according to the different window size and the CNN configurations. One of the important aspects of 
CNN training is feature map weight sharing and how max-pooling layers can reduce the problem to higher and higher levels of important features from the input image. However, the time required to train $\mathrm{CNNs}$ increases when we increase the window size and number of training epochs. We have trained and evaluated $\mathrm{CNNs}$ on a range different epochs $(1,25,50,100$, and 150) and different window sizes.

\section{Visualising output images from training and testing:}

The total number of pixels in the training and testing datasets is 16000 . These pixels are randomly distributed with respect to their $x-y$ coordinates in the input image. The output image after passing the training and testing data through a CNN can be created respect to the $x-y$ coordinates for each pixel, as described in Fig. 4 below.

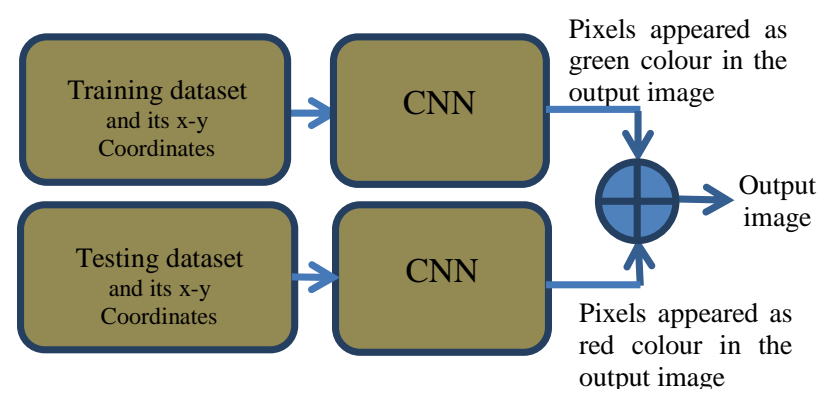

Fig.4. illustrates output image from training and testing dataset

\section{E. Performance measure}

In addition to qualitatively observing the content of output images derived using the process from Fig 4, we can quantitatively evaluate the quality of each output image. We chose Matthews Correlation Coefficient (MCC) as a metric tool when tuning window size for the best CNN configuration. $\mathrm{MCC}$ is a good metric for unbalanced classification datasets. The MCC takes values $[-1,+1]$, where: 1 indicates the absolute correlation between output image and ground truth image when MCC is 0 that means no correlation and; when there is a negative correlation the MCC is -1 . MCC can be calculated using the following equation:

$\mathrm{MCC}=\frac{\mathrm{Tp} * \mathrm{Tn}-\mathrm{Fp} * \mathrm{Fn}}{\sqrt{(\mathrm{Tp}+\mathrm{Fp})(\mathrm{Tp}+\mathrm{Fn})(\mathrm{Tn}+\mathrm{Fp})(\mathrm{Tn}+\mathrm{Fn})}}$

Where, $\mathrm{Tp}$ is True positive, $\mathrm{Tn}$ is True negative, Fp is False positive, and $F n$ is False negative. Tp defines properly detected edge pixels, Fp value gives the number of incorrect edge pixels, Tn pixels is complement to the $\mathrm{Tp}$ and Fn is missing edge pixels. Each of these terms can be calculated by following equations:-

$$
\begin{aligned}
& \mathrm{Tp}=\left|\mathrm{I}_{\text {out }} \cap \mathrm{I}_{\mathrm{GT}}\right| \\
& \mathrm{Fp}=\left|\mathrm{I}_{\text {out }} \cap-\mathrm{I}_{\mathrm{GT}}\right| \\
& \mathrm{Tn}=\left|-\mathrm{I}_{\text {out }} \cap-\mathrm{I}_{\mathrm{GT}}\right|
\end{aligned}
$$

$\mathrm{Fn}=\left|-\mathrm{I}_{\text {out }} \cap \mathrm{I}_{\mathrm{GT}}\right|$

Where, $\mathrm{I}_{\text {out }}$ is output binary image which has edge or notedge pixels, $\mathrm{I}_{\mathrm{GT}}$ is ground truth image, - $\mathrm{I}_{\mathrm{out}}$ is complement of $\mathrm{I}_{\text {out }}$ and - $\mathrm{I}_{\mathrm{GT}}$ is complement of $\mathrm{I}_{\mathrm{GT}}$. [14], [16].

Other valuable quantitative classification metrics are Specificity, Sensitivity and Accuracy [17]. Each of these metrics can be evaluated by the following equations:

Sensitivity $=\frac{\mathrm{Tp}}{\mathrm{Tp}+\mathrm{Fn}}$

Specificity $=\frac{\mathrm{Tn}}{\mathrm{Tn}+\mathrm{Fp}}$

Accuracy $=\frac{T p+T n}{T p+F p+T n+F n}$

\section{F. Final Validation using previously unseen MUI}

For each of the separate CGT and EGT trained/tested CNNs we used three samples of previously unseen ultrasound images for validation. Each $\mathrm{CNN}$ was evaluated using CGT data EGT image data respectively.

\section{EXPERIMENTAL RESULTS}

Our work has been inspired by the recent work of Ciresan et al where CNNs were used to analyse images obtained from electron microscopy [8], but our work uses another biomedical image source: Musculoskeletal Ultrasound Images. In this work, datasets have been prepared for training a CNN and testing (see Fig. 3) We used different input window sizes from 5 to 95 pixels, different $\mathrm{CNN}$ configurations and a suitable epoch number to illustrate which one of these properties support CNN to get the highest level of performance.

We found that the best $\mathrm{CNN}$ performance occurs after 100 training epochs. Moreover, regarding tuning of window size, in the case of using CGT image the best window size was 13 , whilst the best window size was 27 when training CNN on EGT image. The selection of the best window size in this work for both ground truth images used the maximum value of MCC as the optimization criterion. The EGT image described ultrasound image edges relatively simply, while CGT discovered very complex, potentially spurious edges, in the image. So, we did not expect to get agreement in the optimization of the window size for the two CNNs. Fig. 6 and Fig. 7 demonstrate that.

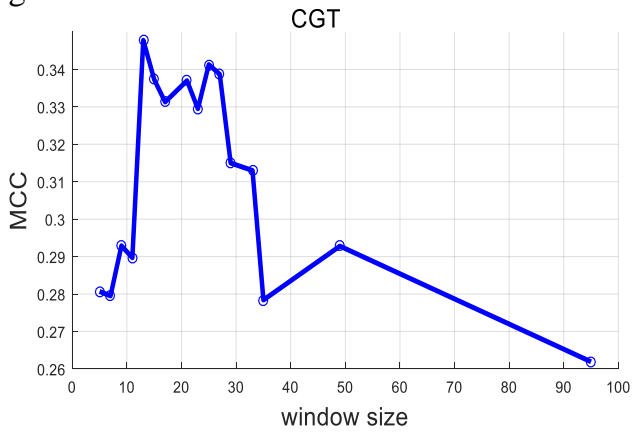

Fig.6. Optimization of window size for CGT 


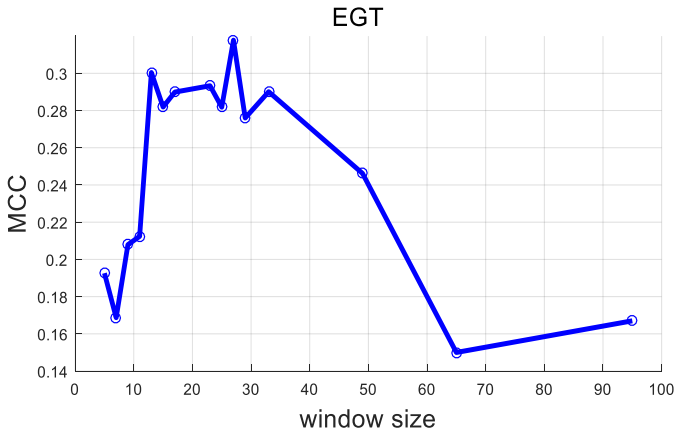

Fig.7. Optimization of window size for EGT image

Table I and Table II illustrates CNN configurations used for both of the above optimal window sizes.

TABLE I

CNN CONFIGURATION (13x13) IN THE CASE OF USING CGT Window size $=13 \times 13$

\begin{tabular}{|c|c|c|c|}
\hline Layer & Type & FM \&Neuron & $\begin{array}{l}\text { Kernel } \\
\text { size }\end{array}$ \\
\hline 1 & Input layer & 1Map of $13 \times 13$ neurons & - \\
\hline 2 & Convolutional & 6 Maps of $10 \times 10$ neurons & $4 \times 4$ \\
\hline 3 & Max-pooling & 6 Maps of $5 \times 5$ neurons & $2 \times 2$ \\
\hline 4 & Convolutional & 12Maps of $2 \times 2$ neurons & $4 \times 4$ \\
\hline 5 & Max-pooling & 12Maps of $1 \times 1$ neurons & $2 \times 2$ \\
\hline 6 & Fully connected & 1neuron & - \\
\hline
\end{tabular}

TABLE II

CNN CONFIGURATION (27x27)IN THE CASE OF USINGEGT Window size $=27 \times 27$

\begin{tabular}{|c|c|c|c|}
\hline layer & Type & FM \&Neuron & $\begin{array}{l}\text { Kernel } \\
\text { size }\end{array}$ \\
\hline 1 & Input layer & 1Map of $27 \times 27$ neurons & - \\
\hline 2 & Convolutional & 6 Maps of $24 \times 24$ neurons & $4 \times 4$ \\
\hline 3 & Max-pooling & 6 Maps of $12 \times 12$ neurons & $2 \times 2$ \\
\hline 4 & Convolutional & 12Maps of $8 \times 8$ neurons & $5 \times 5$ \\
\hline 5 & Max-pooling & 12Maps of $4 \times 4$ neurons & $2 \times 2$ \\
\hline 6 & Convolutional & 12Maps of $2 \times 2$ neurons & $3 \times 3$ \\
\hline 7 & Max-pooling & 12Maps of $1 \times 1$ neurons & $2 \times 2$ \\
\hline 8 & Fully connected & 1neuron & - \\
\hline
\end{tabular}

After identification of the optimal CNN properties (window size, CNN configuration) and training with these properties, $\mathrm{CNN}$ is ready for testing. It is possible to visualize the output image of $\mathrm{CNN}$ from the training and testing processes combined, in the case of using EGT image as example. The process to obtain this image is shown in figure 4. Figure 5 illustrates an output image synthesized from training and testing data shown in different colors.

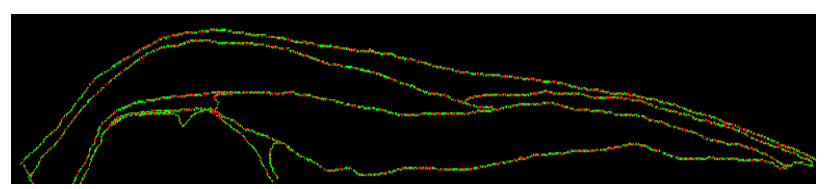

Fig.5. A composite output image produced using the output of a trained CNN showing the training pixels (in green) and the testing pixels (in red) using the data derived from the EGT image.

Of course, in Fig. 5 we see an incomplete image due to selection of just 8000 pixels for training and 8000 pixels for testing, so not all pixels from the original input image are shown (see Fig. 2a).
The evaluation of the two CNN approaches (one using CGT image and the other using EGT image can be succinctly described in the form of two experiments, Experiment 1 and Experiment 2.

Experiment 1

In Experiment 1, Canny ground truth data was obtained by applying a Canny edge-detection operator on the raw input ultrasound image [18]. The Canny operator is traditional method for image edge detection and is automatic since it does not rely on the opinion of an expert in the analysis of MUI. However, we do not expect it to produce the ideal set of edges for our purposes; it is susceptible to noise, and can produce some spurious edges: sometimes extra edges and sometimes missing some important edges.

Figure 8 represents the output image which is obtained from the CGT-trained $\mathrm{CNN}$ when (window size $=13$, epochs $=100$ ).

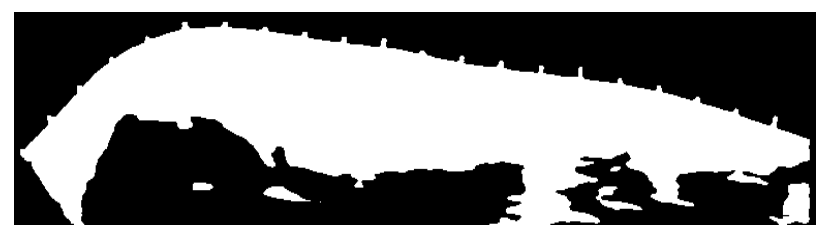

Fig. 8. Output image derived by the CGT-CNN using sample1 as the input image

The CGT-CNN output image above shows us just a full foreground object and its background, but it is impossible to discriminate any boundaries that separate the three most important muscles. The Tp, Fp, Tn and Fn versions of this output image are shown in figure 9. It is clear in the Fp image; there are a lot of error pixels beside real edge pixels, so it is easy to see why all three muscles seem as one full object in figure 8 .
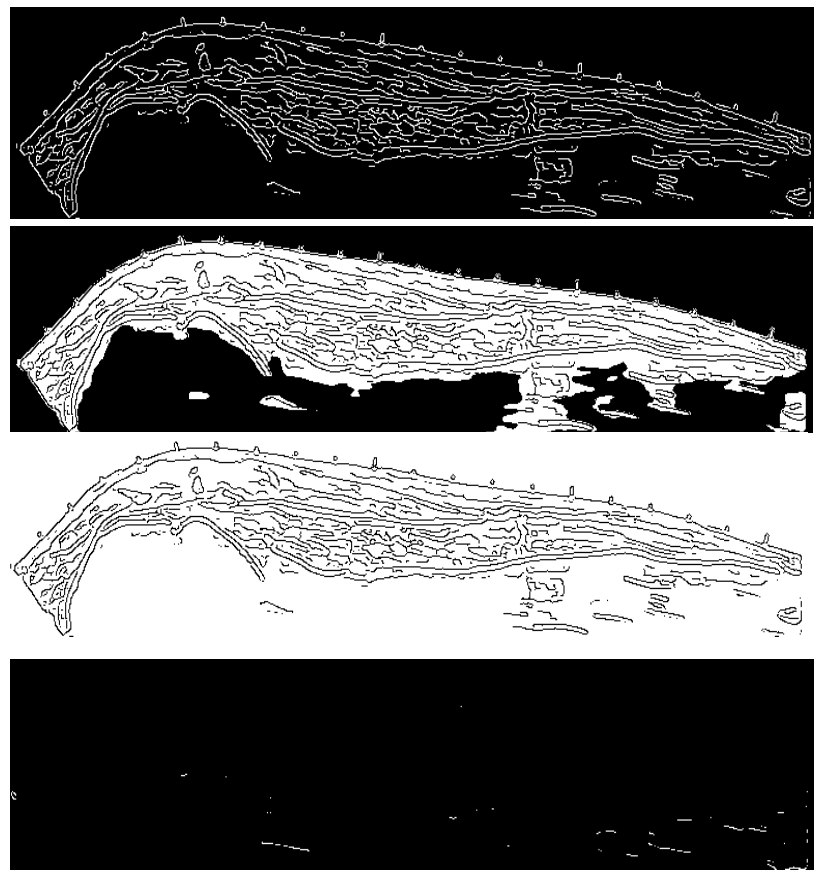

Fig. 9. From top to bottom, Tp, Fp, Tn and Fn of input MUI (sample1) 
Experiment 1 Validation:-

Three unseen MUI images are involved in the validation of this experiment. Figures 10, 11, and 12 below, show the input image (i.e. samples 2, 3 and 4), the CGT for each of the input images and the CGT-CNN derived output images respectively.
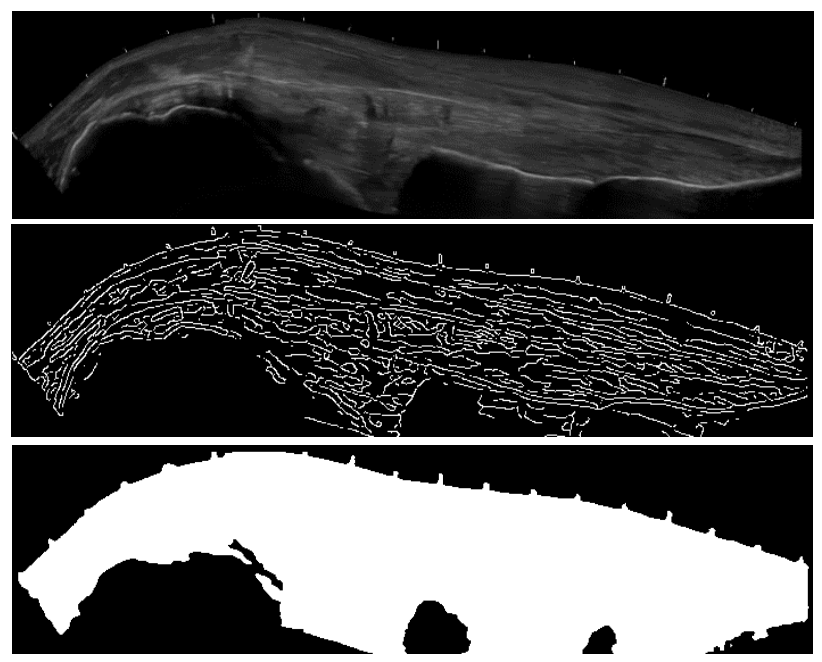

Fig. 10. From top to bottom, input MUI (sample2), CGT and output image.
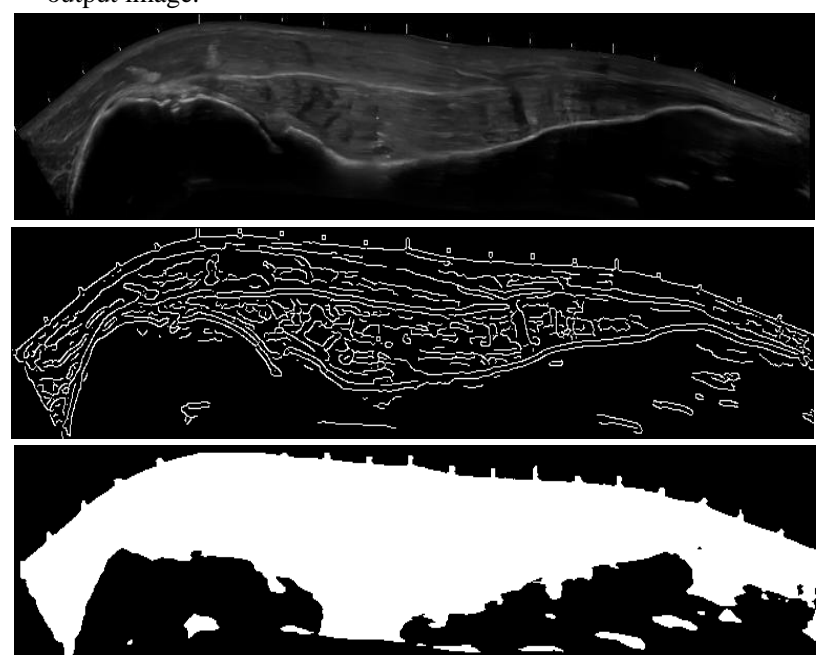

Fig.11. From top to bottom, input MUI (sample3), CGT and output Image.
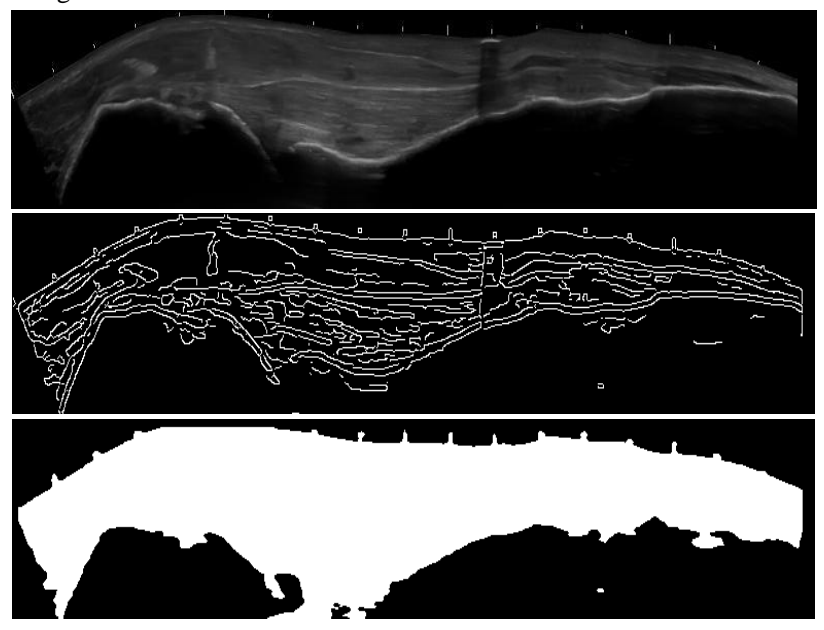

Fig.12.From top to bottom, input MUI (sample4), CGT and output
Experiment 2

The EGT images used for this experiment reflect the expertise of a person who can match anatomical structures with ultrasound imagery. Using the panoramic ultrasound images it is possible to see whole muscles, bones, and tendons. However, using the panoramic technique drawing all of the necessary ground truth images is costly. Furthermore, when gathering EGT data it is difficult to trace the important information of the succession of images and at the same time maintain the necessary alignment between the ground truth image and the original MUI image. However, training an EGT-CNN using this EGT image gives us a clearer set of edges with which to reliably differentiate each of the three muscles. Additionally, if we can train the EGT-CNN on a relatively modest number of images, and have it identify the outlines of muscles with good accuracy; the potential benefits are very large. Figure 13 shows the EGT-CNN output image derived using the sample1 (Fig. 2a) when ( window size $=27$, epochs $=100$ ).

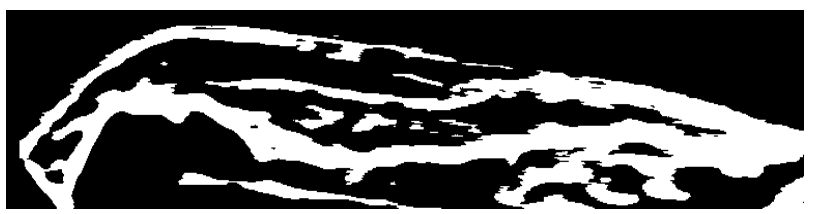

Fig.13. Output image derived by the EGT-CNN using sample1 as the input image

To make a contrast to the experiment 1, Fig. 14 provides us idea about $\mathrm{Tp}, \mathrm{Fp}, \mathrm{Tn}$ and $\mathrm{Fn}$ of the output image in Fig.13, Fp image below tells us there is a statistically significant deference between Fp of this image and Fp in Fig.9. That leads to clear interpretation of image details in the case of training CNN by using EGT image.
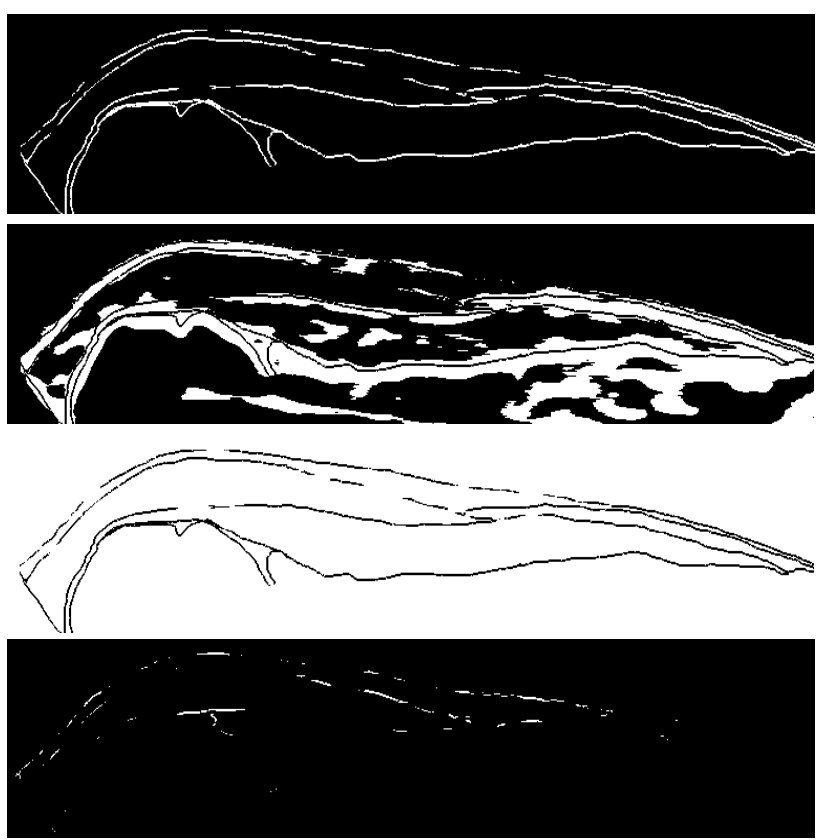

Fig.14. From top to bottom, Tp, Fp, Tn and Fn of input MUI (sample1) 


\section{Experiment 2 Validation}

Again three previously unseen MUI images are involved in this validation. Figures 15, 16, and 17 include input images of the samples $(2,3$, and 4$)$, the equivalent EGT images and the relevant EGT-CNN derived output images, respectively.
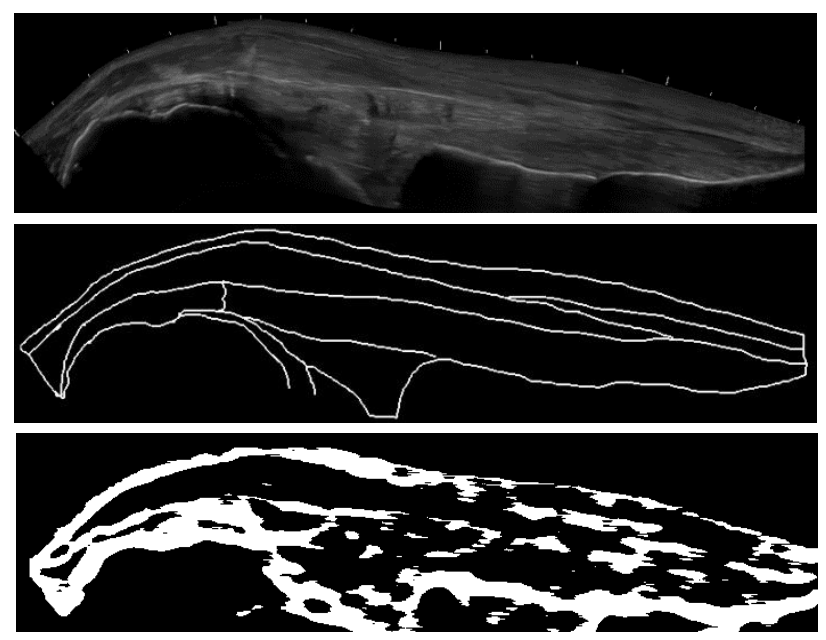

Fig.15. From top to bottom, input MUI (sample2), EGT and output image.
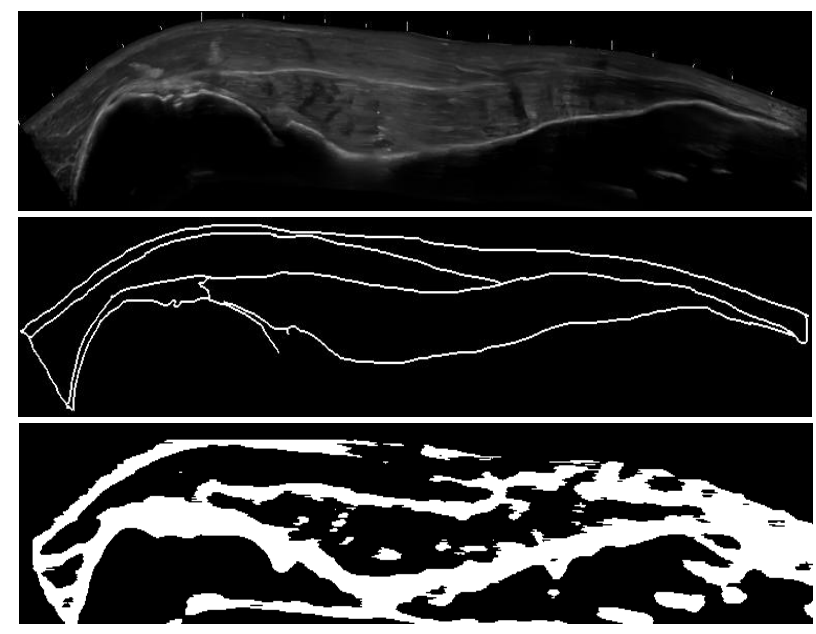

Fig.16. Top to bottom, input MUI (sample 3), EGT and output
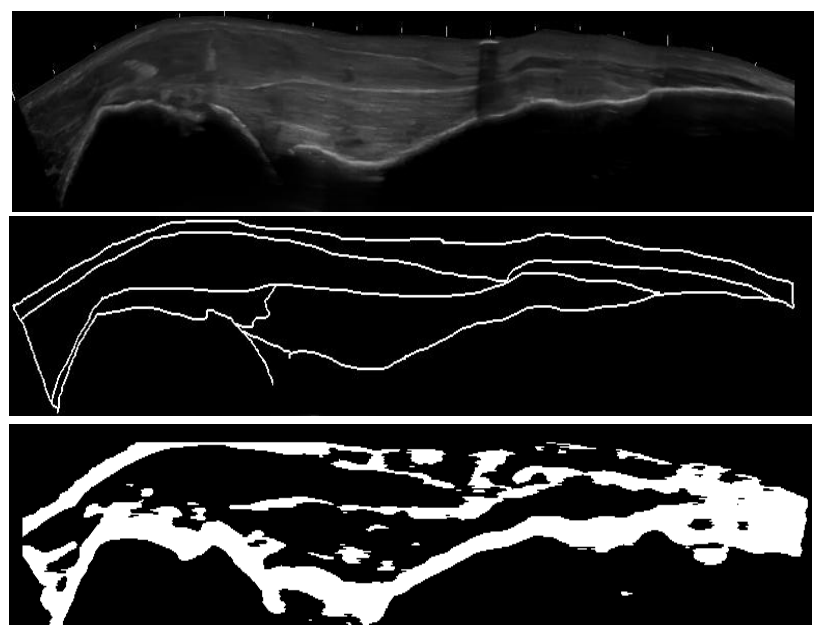

Fig.17. Top to bottom, input MUI (sample 4), EGT and output image.
A quantitative assessment of three previously unseen validation MUI samples obtained from each of the two experiments (including data for the first MUI sample) are shown in table III, below.

TABLE III

QUANTITATIVE ASSESSMENT OF OUTPUT SAMPLES OF MUI

\begin{tabular}{cccc}
\hline \multicolumn{4}{c}{ QUANTITATIVE ASSESSMENT OF OUTPUT SAMPLES OF MUI } \\
\hline \hline Experiment1 & (CGT image) \\
\hline \hline Samples & Accuracy & Specificity & Sensitivity \\
\hline Sample1 & 0.72 & 0.706 & 0.976 \\
Sample2 & 0.67 & 0.65 & 0.99 \\
Sample3 & 0.69 & 0.68 & 0.97 \\
Sample4 & 0.69 & 0.67 & 0.97 \\
Mean & 0.69 & 0.676 & 0.976 \\
\hline Standard & 0.0179 & 0.0202 & 0.0082 \\
deviation & & & \\
\hline \hline & Experiment2 (EGT image) & \\
\hline \hline Sample1 & 0.80 & 0.796 & 0.896 \\
Sample2 & 0.815 & 0.823 & 0.638 \\
Sample3 & 0.786 & 0.787 & 0.766 \\
Sample4 & 0.812 & 0.817 & 0.694 \\
\hline Mean & 0.803 & 0.81 & 0.75 \\
\hline Standard & 0.0132 & 0.0170 & 0.1114 \\
deviation & &
\end{tabular}

\section{IV.DISCUSSION}

From the figures above, in Experiment 2 we can clearly observe the boundaries of three muscles in the Expert Ground Truth (EGT) image derived output images. Whereas, in Experiment 1 the Canny Ground Truth (CGT) derived images make it impossible to see muscle boundaries and instead just a solid foreground object can be separated from the background pixels. This means that qualitatively the output images of CNN by EGT allow better identification of individual muscle than CGT-CNN output images.

In addition Table III gives a quantitative assessment of musculoskeletal image samples for the output from Experiment 1 and Experiment 2. In Experiment 1, our method achieved a lower accuracy value (mean $=0.69$ ) while in the Experiment 2 it is mean $=0.8$ : showing that the EGT-CNN is better able to identify muscle boundaries. Table III also shows a noticeable difference in specificity values between two experiments. The specificity in Experiment 2 is higher than in Experiment 1 because the Experiment 2 output images deliver fewer spurious edge pixels (see figure 9 and figure 14 as example). This indicates that the performance of CNNs trained on EGT image data outperform CNNs trained on CGT images.

According to the equation (3), the number of Fn pixels has a significant impact on the value of the sensitivity. In Experiment 1, the average sensitivity value is high because Fn is perversely very low: and this is due to the output image in this experiment having all of the edge pixels detected in the CGT image, making the resulting sensitivity value approximately equal to $\mathrm{Tp} / \mathrm{Tp}$. However, overall better quality pixel classification is achieved in Experiment 2. Experiment 2's output images give us an 
acceptable way to determine the muscle boundary details of the input. Whereas, the output images of Experiment 1 only allows us to separate foreground objects from the background. The standard deviation of quantitative values in the table III shows us there is little difference between values of all samples.

\section{V.CONCLUSION AND FUTURE WORK}

This initial investigation illustrates that CNN has the potential to do pixel-based edge-detection on Musculoskeletal Ultrasound Images in ways that are akin to a human anatomist with expertise in the analysis of this kind of image. This is a very promising result, firstly because it is the first use of state-of-the-art CNNs that has focused on Musculoskeletal Ultrasound Image edge detection. Secondly, it has the potential to be a scalable solution that could improve the usefulness of important approaches in Musculoskeletal Ultrasound Image such as the panoramic technique.

This paper has reported work that is still in progress and for the future we propose to increase the size of the $\mathrm{CNN}$ training datasets to raise the $\mathrm{CNN}$ performance. Using GPU will help us in accelerating training and reduce implementation time. In addition, so far the current work has not used any kind of pre-processing like foveation [8], contrast enhancement or any active denoising to reduce the effects of speckle noise [4]. If carried out this kind of processing is likely to boost CNN performance, but at the expense of extra pre-processing computation: so a trade-off between these pros and cons still needs to be found to overcome the confusion that can happen due to speckle noise or low contrast. In this work we chose a sigmoid function as the activation function to get a simple threshold which separates two output classes. We plan some refinements that might improve the CNN performance such as changing the activation function to use rectified linear units (ReLUs) instead of sigmoid function. ReLUs have good properties such as unbounded range, and are good at dealing with sparsity and big datasets [15]. Another important issue to be addressed is the wider collection of Musculoskeletal Ultrasound Imagery from healthy persons: because healthy muscle fibers are organized as more uniform patterns and may therefore allow better ultrasound discrimination between muscle tissue and body fat [19].

\section{VI.REFERENCES}

[1] S. Pillen, "Skeletal muscle ultrasound," European Journal Translational Myology, 1(5), 145-155, 2010.

[2] B. Bolsterlee, D.H. Veeger,E. K. Chadwick, "Clinical applications of musculoskeletal modelling for the shoulder and upper limb"International Federation for Medical and Biological Engineering, 51(9),953-963,2013

[3] E. K. Chadwick, D. Blana, R. F. Kirsch, \&, A. J. Van Den Bogert, "Real-Time Simulation of Three-Dimensional Shoulder Girdle and Arm Dynamics"IEEE Transactions on Bio-Medical Engineering, 61(7), 1-10, 2014.

[4] R. Gupta, I. Elamvazuthi, S. Dass, I. Faye, P.Vasant, J. George, \& F. Izza, "Curvelet based automatic segmentation of supraspinatus tendon from ultrasound image: a focused assistive diagnostic method,'BioMedical Engineering Online, 2014.http://www.biomedical-engineering-

online.com/content/13/1/157

[5] F. J. Gallegos-funes, M. Jose, A.J. Rosales-silva \& S.S. Isakina, "Real-Time Speckle and Impulsive Noise Suppression in 3-D Ultrasound Imaging"Ultrasound Imaging, ISBN: 978-953-307-2395, 2011,http://www.intechopen.com

[6] N. Biradar, M. Dewal,\&M. Rohit, "Edge Preserved Speckle Noise Reduction Using Integrated Fuzzy Filters"International Scholarly Research Notices, 1-11, 2014, doi:10.1155/2014/876434.

[7] A.Krizhevsky, \& G. Hinton, "ImageNet Classification with Deep Convolutional Neural Networks,"Advances in Neural Information Processingsystems Conference, 1097-1105, 2012.

[8] D. Ciresan, A. Giusti\& L. M. Gambardella,"Deep Neural Networks Segment Neuronal Membranes in Electron Microscopy Images",NIPS, 2852-2860, 2012.

[9] D. Ciresan, A. Giusti\& L. M. Gambardella, "Mitosis Detection in Breast Cancer Histology Images with Deep Neural Networks,"MICCAI,2, 411-418, 2013.

[10] H.Wang, A. Cruz-roa,A. Basavanhally,H. Gilmore,N. Shih,M. Feldman, J. Tomaszewski,F. Gonzalez and A. Madabhushi,“ Mitosis detection in breast cancer pathology images by combining handcrafted and convolutional neural network features,"Journal of Medical Imaging, 1(3), 2014.

[11] C. Cernazanu-glavan, \& S. Holban,"Segmentation of bone structure in X-ray images using convolutional neural network. Advances in Electrical and Computer Engineering," 10(1), 1-8, 2013.

[12] M. Melinscak, P. Prentasic, \& S. Loncaric, "Retinal Vessel Segmentation Using Deep Neural Networks,"10 International Conference on Computer Vision Theory and Applications, pp. 577$582,2015$.

[13] Y.LeCun, L.Bottou, L.Bengio, and P.Haffner, "Gradient-Based learning applied to document recognition," Proceedings of the IEEE, 86(11),2278-2324,1998.

[14] K.Özkan, "A novel multi-scale and multi-expert edge detector based on common vector approach,"International Journal of Electronics and Communications, 69, 1272-1281,2015.

[15] J. Doorn, "Analysis of Deep Convolutional Neural Network Architectures," $21^{\text {th }}$ Twente Student Conference on IT,University of Twente, Netherlands, 2014.

[16] B.Matthews, "Comparison of the predicted and Observed secondary structure of T4 Phage Lysozyme", Biochimica et Biophysica Acta, 405, 442-451,1975.

[17] C. Lopez-Molina, B. De Baets, \& H. Bustince, "Quantitative error measures for edge detection,"Pattern Recognition, 46(4), 1125$1139,2013$.

[18] J.Canny, "A Computational Approach to Edge Detection,"IEEE Transactions on pattern analysis Machine Intelligence, 8(6), 679698, 1986.

[19] PK. Nielsen , BR Jensen , T. Darvann , K.Jørgensen , M. Bakke, "Quantitative ultrasound image analysis of the supraspinatus muscle," ClinicalBiomechanical, 15(Supplement 1(0)):S13$\mathrm{S} 16,2000$. 